\title{
DEVELOPMENT OF A 7000 x 4000 PIXEL MOSAIC CCD CAMERA
}

Nobunari Kashikawa, Masafumi Yagi, Naoki Yasuda, Sadanori Okamura, Kazuhiro Shimasaku, Mamoru Doi and

University of Tokyo, Bunkyo-ku

Maki Sekiguchi

National Astronomical Observatory of Japan

\section{CCD CHIPS, MOUNTING PROCEDURES, MECHANICAL \& ELECTRIC DESIGN}

The CCD we use is TC215 manufactured by TI, Japan. The pixel size is 12 microns square. It is a virtual phase CCD which has a peak QE of $60 \%$ at $700 \mathrm{~nm}$ and $15 \%$ QE at 350 $\mathrm{nm}$. It is commercially available in a package, which is too big to meet our requirement for CCD spacing. We therefore put the CCD in a specially made compact package. Each chip is mounted on a machined ceramic spacer whose thermal coefficient is matched with that of the CCD package. We glue each CCD chip on the spacer under a microscope to measure YHE $\mathrm{x}$ $y$ position and height. Then we screw the CCD chip + spacer on a copper motherboard with the help of the gauge which has a planned grid with a good accuracy. The alignment accuracy we can get with such set-up procedures is an order of 5 microns $(<0.5$ pixel $)$ in the horizontal direction and 10 microns in the vertical direction.

The CCDs are put in a dewar of the size $30 \mathrm{~cm} \times 30 \mathrm{~cm} \times 10 \mathrm{~cm}$ (WDH) with a window optimized for an eight by four array (See Fig.1.). A large focal plane shutter and a filter holder are attached in front of the dewar. It takes about eight seconds for the shutter to fully open/close. The shutter has two bellows on each side, and the exposure time is kept uniform over the chips by moving the two bellows in appropriate turns. We have made four large (20 $\mathrm{cm} \times 20 \mathrm{~cm}$ ) filters whose responses are close to those of Johnson-Mould's B, V, R and I bands. The CCDs are cooled down to $-80^{\circ}$ to $-90^{\circ}$ by both a Stirling-cycle cooler and liquid nitrogen. The vibration caused by a compressor of the cooler is confirmed to be negligible for a 12 micron pixel. A liquid nitrogen tank is necessary, not only as a cooler, but also as a cryogenic pump. We use a system called MESSIA2 (Modularized Expandable SyStem for Image Acquisition) for CCD control and data acquisition. Four columns, each consisting of seven CCDs, are read in parallel, while in each column seven CCDs are read out one-by-one serially. The total CCD readout time, i.e., the time necessary to read CCDs, digitize, and store the data in the VME memory boards located near the camera, is about 100 seconds. Once the data are stored in VME memory boards, the next exposure can be started. The data are transferred to hard disks of a control computer during the exposure.

\section{SOFTWARE, PERFORMANCE AND PROSPECTS}

The data produced by the camera amount to about 1 GB per night. Software which 


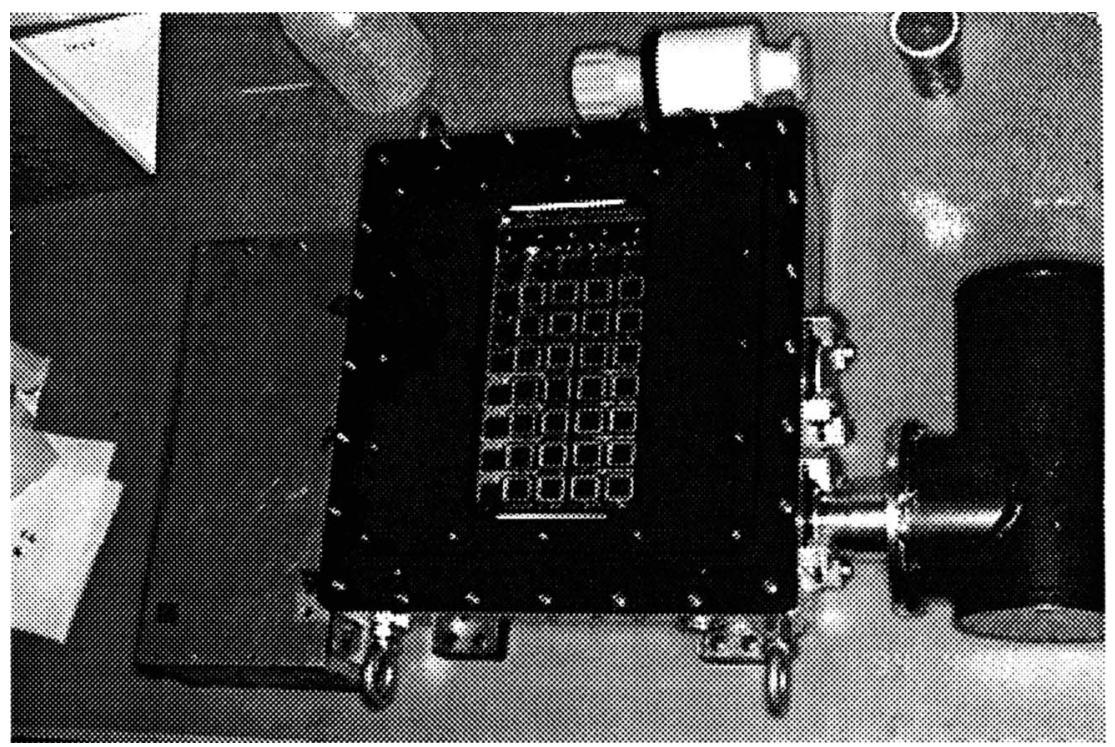

Fig. 1. The dewar of the mosaic CCD camera developed by the Univ. Tokyo-NAOJ group. 7 by $4=28$ CCDs are mounted. A liquid nitrogen tank is on the left and on the right is a Stirling cooler.

reduces this vast amount of data has also been developed. The data reduction system consists of several modules, which do specific tasks such as flatfielding, sky subtraction, find and measure objects, measure seeing size, match objects in overlapped regions, star-galaxy separation, and crude morphological classification of galaxies. The system also has functions to plot variety of statistical quantities in many kinds of graphs for an easy and quick monitoring of whether or not the system is processing the data in a proper way. The best performance of the system is realized by two-pass processing. The first pass is to fine-tune several critical parameters that are used in the second pass, which produces the final catalog of objects with photometric parameters together with parameters necessary to produce one contiguous image from $28 \times 4$ $=112$ frames.

We have not yet completely finished evaluating all the performances, which are expected to be the same as or better than those of MCCD1 (Sekiguchi et al. 1992). Our primary motivation for these cameras is observational cosmology based on wide field surveys. We need a wide-field telescope at a good site in order to take full advantage of MCCD2. In collaboration with Carnegie Institution we used MCCD2 in May 1994 with the 40-inch telescope at Las Campanas Observatory to take multi-color images of southern clusters of galaxies. MCCD2 with the Swope telescope give an image scale of $0.35 \mathrm{arcsec} / \mathrm{pixel}$ and a sky coverage of $74 \times 43$ arcmin with four exposures. More observations are planned in Oct. - Nov. 1994. Our goal is to expand the array to eight by eight CCDs, which would be a sort of prototype of the prime-focus camera for our Japanese 8-m telescope (SUBARU).

\section{REFERENCE}

Sekiguchi, M., Iwashita, H., Doi, M., Kashikawa, N. and Okamura, S.. 1992 PASP 104, 744 